\title{
Model for Nanofluids Thermal Conductivity Based on Modified Nanoconvective Mechanism
}

\author{
Yuant Tiandho ${ }^{1,2^{*}}$, Rika Favoria Gusa ${ }^{1,3}$, Irwan Dinata ${ }^{1,3}$ and Wahri Sunanda ${ }^{1,3}$ \\ ${ }^{1}$ Research Center for Energy and Information Technology, Universitas Bangka Belitung, Bangka - Indonesia \\ ${ }^{2}$ Department of Physics, Universitas Bangka Belitung, Bangka - Indonesia \\ ${ }^{3}$ Department of Electrical Engineering, Universitas Bangka Belitung, Bangka - Indonesia
}

\begin{abstract}
Nuclear reactors are one of the long-term energy fulfillment solutions. Efforts to increase operating power density at various type of nuclear reactors are programs that are being developed to improve the economic properties of a reactor. The use of nanofluids allows nuclear reactors to operate more optimally through increased critical heat flux (CHF) and increased retention capability of nuclear reactors to accidents. Thermal conductivity is a nanofluids property that intensively studied because it has not been obtained an accurate model. In this paper a nanofluid thermal conductivity model was developed by involving all possible heat transfer mechanisms. But the modification only focuses on the mechanism of nanoconvection. According to this model the thermal conductivity of nanofluids depends on the volume fraction of nanoparticles, particle diameter, viscosity, density, and temperature.
\end{abstract}

Keywords: Nanofluids; thermal conductivity; nuclear reactor; nanoconvective.

\section{Introduction}

The urgency of reducing carbon emissions from various energy sources in the world is increasingly emphasized to avoid increasing the impact of climate change. Suggestion regarding the target of reducing global emissions to $<1$ trillion tons of $\mathrm{CO}_{2}$ in 2100 become a focus on various sectors in the world. Therefore, many countries have developed various technologies of zero carbon emission sources. One of the energy fulfillment technologies with the lowest carbon emission level is nuclear power. Development of nuclear reactor technology is still being developed massively to satisfy its security requirements and economic aspects [1].

Currently, efforts to increase the operating power density of light water reactor (LWR) is one of the approaches used to improve the economic properties of a reactor. There are several possible attempts. One interesting alternative effort besides conventional efforts such as fuel assembly management and optimization fuel design engineering is the seeding of cooling water with nanoparticles to produce fluids with superior heat dissipation [2]. Several tests at MIT's Nuclear Research Reactor have shown that the use of nanofluids (i.e. fluids with dispersed nanoparticles) can increase the productivity of pressurized water reactor (PWR). Nanofluids can prevent the formation of a vapor layer around the fuel rods and it can increase critical heat flux (CHF) significantly [3]. The increasing of CHF in nanofluids with nanoparticles such as silicon, aluminum, and titanium oxide can reach $200 \%$ [4]. In addition, the retention of nuclear reactors increased by $40 \%$ when accidents on the use of nanofluids was a very promising prospect $[5,6]$.

The ability of heat transfer is one of the most interesting aspects of nanofluids [7]. Some theories related to heat transfer mechanisms such as the Maxwell suspension approach, heat propagation speed [8], and Brownian analysis on nanofluids [9] are some theories that are widely used to explain the heat characteristics of nanofluids. Nevertheless, the theory has not yet been obtained a satisfactory description.

The nanofluids heat transfer mechanism proposed by Jang and Choi [7,9] has a complex picture. In the model are presented various possible heat transfer mechanisms such as conduction by intermediate base fluid, internanoparticles, and convection between fluid base with nanoparticles. However, in the proposed model there are several approaches such as the expression of the Nusselt number as the quadratic function of the Reynolds and Prandtl numbers which are often not appropriate for some conditions [10-12]. Furthermore, the study of the viscosity dependence of nanofluids on temperature has not been explicitly described in the model. Whereas viscosity is a parameter that gives a significant influence on convection coefficient of nanofluids [13]. Therefore, in order to correct these problems, we propose a modification of the nanofluids conductivity model especially for the nanoconvention aspect.

\section{Modelling the Nanofluids Thermal Conductivity}

In the model constructed by Jang and Choi [7] it is stated that basically the conductivity of nanofluids can be

* Corresponding author: yuant@ubb.ac.id 
analyzed based on four mechanisms. The first mechanism is the heat transfer mechanism generated by the collisions between the fluid base molecules. The second mechanism is generated from the thermal diffusion of the nanoparticles in the fluid. The third mechanism is derived from collisions between nanoparticles due to Brownian motion. However, based on calculations, the energy flux value in the third mechanism is not significant and this mechanism is often neglected. While the last mechanism is a thermal interaction of the dynamics or dancing of nanoparticles with a fluid base molecule, also called the nanoconvection mechanism.

Basically in thermal conduction analysis it always involves the transfer of energy from particles that have higher thermal energy to particles that have lower thermal energy due to the interaction between particles. In gases and liquids, the thermal energy can be transferred through the interaction of collisions between particles. Based on the kinetic theory, the net energy flux passing through the $z$-plane is given by [7],

$$
J_{v}=\left[\rho_{v}(z)-\rho_{v}(z+l)\right] \bar{C}=-l \hat{C}_{V} \bar{C} \frac{d T}{d z}
$$

where $J_{v}, \bar{C}, \hat{C}_{V}, l, T$, and $\rho_{v}$ are energy flux, molecular average velocity, heat capacity per unit volume, mean free path, temperature and energy density respectively. The spatial averages of these are [14]. Thus the thermal conductivity is given by,

$$
k=\frac{1}{3} l \bar{C} \hat{C}_{V}
$$

In the first heat transfer mechanism because it involves only collisions between the fluid base molecules and if there is a fraction of nanoparticles in the fluid, $f$, then the net flux is,

$$
J_{v 1}=-\frac{1}{3} l_{B F} \hat{C}_{V, B F} \bar{C}_{B F}(1-f) \frac{d T}{d z}=-k_{B F}(1-f) \frac{d T}{d z}
$$

where $k$ is the thermal conductivity and a parameter with subscript $B F$ related to physical parameter for base fluid. Furthermore it can easily defined the net flux due to the second mechanism as,

$$
J_{v 2}=-\frac{1}{3} l_{p} \hat{C}_{V, P} \bar{C}_{P} f \frac{d T}{d z}=-k_{p} f \frac{d T}{d z}
$$

where a parameter with subscript $P$ related to physical parameter for nanoparticle. However in the second mechanism there is an obstacle that interrupt the heat transfer process called the Kapitza resistance factor thus the nanoparticle conductivity of the second mechanism can be expressed as,

$$
k_{P}=\beta k_{P o}
$$

where $k_{P o}$ is the conductivity of the particle before it involves the Kapitza resistance. The third mechanism involving collisions between nanoparticles due to Brownian motion and it produces energy flux as,

$$
J_{v 3}=-\frac{1}{3} \Lambda_{P} \hat{C}_{V, P} \bar{C}_{T} f \frac{d T}{d z}
$$

where $\Lambda_{P}$ and $\bar{C}_{V}$ are the mean collision length of particles and the translational velocity of particles. Through the kinetic theory the translational velocity can be expressed as $\bar{C}_{T}=\sqrt{\frac{18 k_{b} T}{\rho \pi d_{P}^{3}}}$ where $K_{B}$ is Boltzman

constant. From the formula it can be found that the energy flux range is at the order of $10^{-5}$ and the value is much smaller than other mechanisms [15]. Therefore in this paper the third mechanism is not taken into account. In the last mechanism, i.e. the nanoconvective mechanism, the energy flux in the heat transfer process can be defined as,

$$
J_{v 4}=h\left(T_{P}-T_{B F}\right) f=h f \delta_{T} \frac{\left(T_{P}-T_{B F}\right)}{\delta_{T}} \sim-h f \delta_{T} \frac{d T}{d z}
$$

where $h$ and $\delta_{T}$ are convection heat transfer coefficient and thickness of the boundary layer respectively. Thus without involving the third mechanism, the effective thermal conductivity $\left(k_{e f f}\right)$ of the nanofluids will lead to,

$$
k_{e f f}=k_{B F}(1-f)+\beta k_{P} f+h f \delta_{T}
$$

In nanoscale, thermal boundary layer will reduce to Prandtl number relation,

$$
\delta_{T} \sim \frac{\delta}{\operatorname{Pr}}
$$

Where $\delta$ is the hydrodynamic boundary layer. In Jang and Choi's model [7] it is postulated that nanolayers of ordered liquid molecules act as hydrodynamic boundary layers and it expressed as,

$$
\delta \sim A d_{B F}
$$

where $\mathrm{A}$ is a proportional constant and in literature the value is $A=18 \times 10^{6}[7,9]$. In the heat transfer to a surface in the fluid, the Nusselt number $(N u)$ describes the ratio of convection heat transfer to conduction over the surface. Hence the Nusselt number is often used as a bridge to explain the convection coefficient in conduction heat transfer frames. In general the Nusselt number is defined as,

$$
N u=\frac{h D}{k}
$$

In the nanoscale case, the characteristic length of nanofluids, $D$, can be defined as the nanoparticle equivalent diameter and $k$ is $k_{B F}$ [9]. However in a macroscopic view with the interaction between nanofluids and fluid flow geometry the characteristic length can be expressed as the hydraulic diameter of channel while the parameter $k$ in eq. (11) is expressed as $k_{\text {eff }}[16,17]$. Thus for this article the expression of the convective coefficient can be written as

$$
h=\frac{k_{B F} N u}{d_{p}}
$$

Heat transport in nanofluids systems has the same characteristics as heat transfer in fluidized beads. Brodkey et al. [17] have reported that fluidized beds (contain micron-sized particles) have Nusselt numbers that are 20100 times larger than single particles. Therefore, to satisfy these conditions, based on the relationship between Nusselt numbers for particles on heat transfer fluidized beds, the expression h must be modified [19]. In general, the Nusselt number is a function that contains [20,21],

$$
N u=N u\left[\operatorname{Re}, \operatorname{Pr}, K_{B F}, K_{p}, f \text {, particle shape }\right]
$$


where $R e$ is the Reynolds number. Currently there are many Nusselt number expressions that have been used as in Table 1. Altough almost all Nusselt number expressions contain parameters of Reynolds and Prandtl numbers and almost all of them satisfy the expression of the Nusselt number function as eq. (13) but these expressions contain significantly different numbers of Reynolds and Prandtl numbers. Therefore declaring the $k_{\text {eff }}$ formula as a function of the Reynolds number and Prandtl number explicitly through the Nusselt number expression reduction is quite complicated. There are many possible combinations allowed. Except by the expression of the Nusselt number proposed by Zerradi [12] which states the Reynolds number and Prandtl number as the termophysical coefficient and it depends on the experimental data fitting.

Table 1. Definition of Nusselt number in the literatures

\begin{tabular}{cl}
\hline Ref. & Nusselt Number \\
\hline [22] & $N u=2+0.5 \operatorname{Re} \operatorname{Pr}$ \\
[9] & $N u=\operatorname{Re}^{2} \operatorname{Pr}^{2}$ \\
{$[11]$} & $N u=0.021 \operatorname{Re}^{0.5} \operatorname{Pr}^{0.5}$ \\
& $N u=0.0059\left(1+7.6286 f^{0.6886}\left(\operatorname{Re} \operatorname{Pr} \frac{d_{p}}{D}\right)^{0.001}\right)$ \\
{$[10]$} & $\times \operatorname{Re}^{0.9238} \operatorname{Pr}^{0.4}$ \\
& $N u=\operatorname{Pr}^{p}\left(\alpha f+\gamma \operatorname{Re}_{m}^{q}+\chi f \operatorname{Re}_{m}^{q}+\delta\right)$ where the
\end{tabular}

[12] Greek symbols correspond to thermophysical coefficient

One possible alternative to solve the difference problem in determining the Nusselt number is still to declare the $k_{\text {eff }}$ to be explicitly dependent on the Nusselt number. The nanofluids conductivity remains presented in eq. (15) and does not need to expand it to the expression of the Reynolds number and the Prandtl number. While the experimental value of Nusselt number of a fluid can be determined through the instrument system [23]. However various studies have reported that the Nusselt number continues to change with changing rheological factors. Thus it will create another problem. Therefore, to avoid the inconsistency of the formula with experimental data it is worthy to declare the Nusselt number as a function of the termophysical coefficient as in Zerradi model [12].

Using the fluidized beds analogy to the general relation of the convection coefficient for Brownian motion-induced Stokes flow of multiple nanoparticles has a modified form as,

$$
h_{m}=\frac{k_{B F}}{d_{p}}\left(C \operatorname{Re}^{m} \operatorname{Pr}^{n} f\right)
$$

Through the substitution of eq. (11), (9), and (10) into eq. (8) it will lead us to,

$$
\frac{k_{e f f}}{k_{B F}}=(1-f)+\beta \frac{k_{P}}{k_{B F}} f+\frac{D d_{B F}}{d_{p} \operatorname{Pr}}\left(\operatorname{Re}^{m} \operatorname{Pr}^{n} f\right)
$$

In Fig. 1 a comparison between models in eq. (15) with the experiments by Mintsa et. al [24] is shown. We compared the model on two types of nanofluids i.e. $\mathrm{CuO}$ and $\mathrm{Al}_{2} \mathrm{O}_{3}$ which were dispersed in water. The material properties of the fluid and nanoparticles used we refer to Jang \& Choi experiments [7]. It appears the model we proposed is quite appropriate with the experimental data because the variance is small. From the results it can be stated that the volume fraction of the dispersed nanoparticles in the fluid directly proportional with the conductivity. For information, through fitting the data can be obtained the parameters of thermophysical parameters: $m$ and $n$. For $\mathrm{CuO}$ the value $m=1.257$ and $n=1$, while for $\mathrm{Al}_{2} \mathrm{O}_{3}$ the value $m=1.576$ and $n=1$. Based on the results it appears that the value of the thermophysical parameters obtained through this model is lower than the model proposed by Jang and Choi [7] but higher than that the model proposed by Pak and Cho [11]. In addition, it is clear that the thermophysical parameters for different nanofluids may have different values. Thus declaring those thermophysical parameters in a constant will decrease the accuracy of the proposed model.

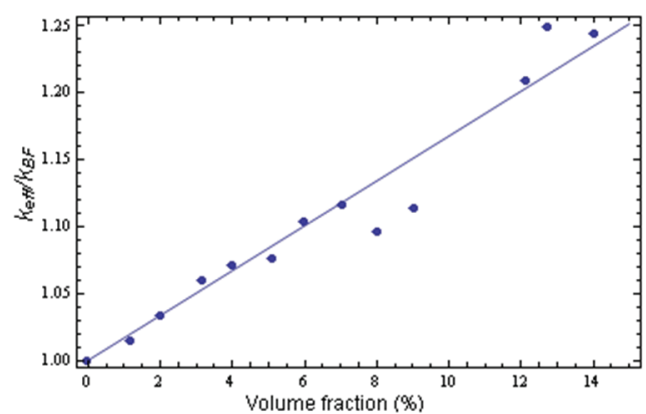

(a)

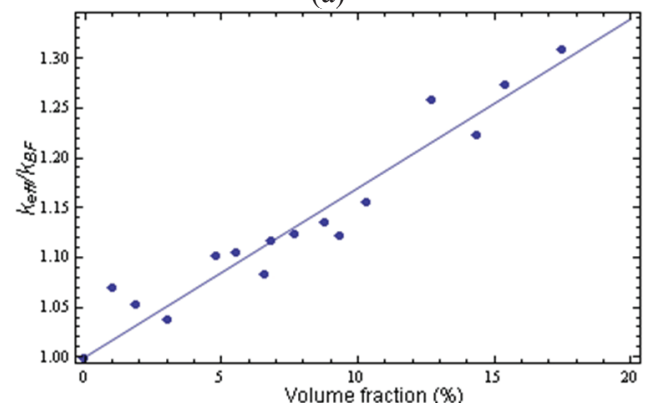

(b)

Figure 1. Comparison of the model in eq. (15) with the experiment [24] for $k_{\text {eff }} / k_{B F}$ in nanofluids (water based): (a) $\mathrm{CuO}$ with $d=29 \mathrm{~nm}$ and (b) $\mathrm{Al}_{2} \mathrm{O}_{3}$ with $d=36 \mathrm{~nm}$.

Other interesting results that can be found in $\mathrm{CuO}$ and $\mathrm{Al}_{2} \mathrm{O}_{3}$ nanofluids the both Prandtl numbers are almost same. Physically, the Prandtl number represents the momentum diffusivity ratio to the thermal diffusivity that is,

$$
\operatorname{Pr}=\frac{c_{p} \mu}{k}
$$

where $c_{p}$ and $\mu$ are heat type and dynamic viscosity respectively. In nanofluids due to occurs interaction between nanoparticles, fluid base, or both, the physical parameter of the above equations can be viewed as effective conditions. The effective specific heat of nanofluids can be expressed as [25], 


$$
c_{p, e f f}=\frac{(1-f) \rho_{B F} c_{p, B F}+f \rho_{p} c_{p, \mathrm{p}}}{\rho_{\text {eff }}}
$$

While the effective density of nanofluids can be determined from the equation,

$$
\rho_{\text {eff }}=(1-f) \rho_{B F}+f \rho_{p}
$$

The effective viscosity of nanofluids as a function of volume fraction nanoparticles is defined as,

$$
\mu_{\text {eff }}=\mu_{B F}+\frac{\rho_{p} V_{B} d_{p}^{2}}{72 F \delta}
$$

While $V_{B}$ is Brownian velocity, $V_{B}=\frac{1}{d_{p}} \sqrt{\frac{18 k_{b} T}{\pi \rho_{p} d_{p}}}, \delta$ is the distance between particles, $\delta=\sqrt{\frac{\pi}{6 \phi}} d_{p}$, and $F$ is correction factor correspond to the volume fraction and the average diameter of nanoparticles. Looking at the expression of the above number, it is safe to say the Prandtl number reduce to unity. Because in the case of $\mathrm{CuO}$ and $\mathrm{Al}_{2} \mathrm{O}_{3}$ an increase in specific heat value is also followed by an increase in its conductivity. It has also frequently used Prandtl numbers of unity on a variety of nanofluids parameter analysis although in extreme conditions the Prandtl number can reach 2000 when its Reynolds number revolves around the order of $10^{6}$ [26].

Temperature is a physical parameter that can modify the viscosity of a fluid. This aspect is important because the viscosity also contributes significantly to the Reynolds number. Through the comparison between the Reynolds model and the Vogel model, in this paper the viscosity value as a function of temperature is expressed as,

$$
\mu(T)=\mu_{0}+\mu_{1} \exp (-T / a)
$$

where, $\mu_{0}, \mu_{1}$, and $a$ are parameter whose value depends on the type of nanoparticles and fluids. Fig. 2 shows the comparation between the model we proposed with the viscosity value as a function of temperature for waterbased nanofluids: $\mathrm{CuO}$ and $\mathrm{Al}_{2} \mathrm{O}_{3}$ [13]. Through the comparison it appears that the viscosity model as a function of the temperature is quite in accordance with the experimental results. In addition, the accuracy of this model is higher when compared with the various models that have been studied by Masoumi et. al [13].

According to MacLaurin series the viscosity expression in eq. (2) can written as,

$$
\mu(T)=\mu_{1}\left(\left(1+\mu_{0}\right)-\frac{T}{a}+O\left(T^{2}\right)\right)
$$

Basically eq. (21) can be expressed as a general expression of the relationship between viscosity and temperature as reported by Nguyen et. al. In the measurement Nguyen obtained a viscosity relationship as a function of temperature for nanofluids with concentrations of $1 \%$ and $4 \%$ respectively as [27],

$$
\begin{gathered}
\mu_{e f f}=\mu_{B F}(1.125-0.0007 T) \\
\mu_{e f f}=\mu_{B F}\left(2.1275-0.0215 T+0.0002 T^{2}\right)
\end{gathered}
$$

The definition of Reynolds numbers in nanofluids can be expressed as [8],

$$
\operatorname{Re}=\frac{G C_{R M} d_{p}}{v}
$$

where $C_{R M}, v$, and $G$ are velocity of random motion of nanoparticles, kinematic viscosity, and correction factor. The definition of velocity of random motion can be obtained through the relation between macroscopic diffusion coefficient $D_{0}$ with the mean free path of base fluid, $l_{B F}$ as,

$$
C_{R M}=\frac{D_{0}}{l_{B F}}
$$

and the nanoparticles diffusion coefficient given by Einstein as, $D_{0}=k_{b} T / 3 \pi \mu d_{p}$ [27].

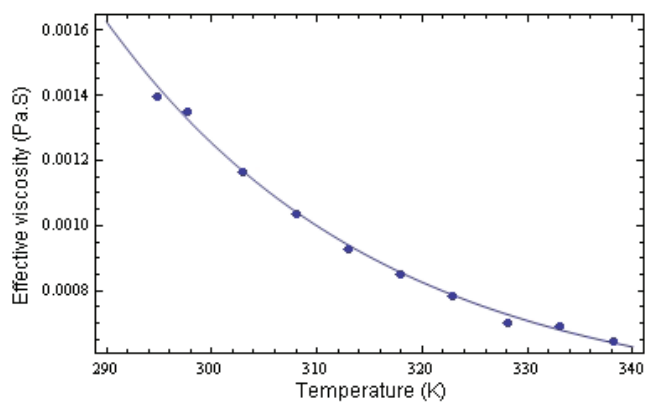

(a)

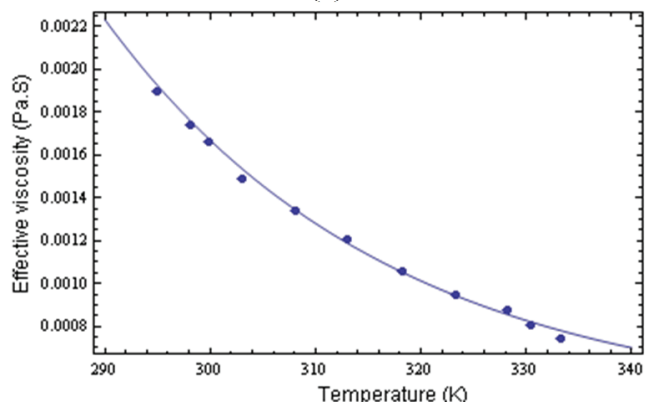

(b)

Figure 2. Comparison of viscosity model in eq. (20) with experiment result [13] for water-based nanofluids: (a) $\mathrm{Al}_{2} \mathrm{O}_{3}(d$ $=36 \mathrm{~nm}, f=4.5 \%)$ and (b) $\mathrm{CuO}(d=29 \mathrm{~nm}, f=4.5 \%)$.

Through the relationship between kinematic and dynamic viscosity for its effective value based on eq. (21) it lead us to the relationship of conductivity of nanofluids as temperature function. Assuming the value of Prandtl number is one the expression of nanofluids conductivity is,

$$
\frac{k_{\text {eff }}}{k_{B F}}=(1-f)+\beta \frac{k_{p}}{k_{B F}} f+\frac{D d_{B F} f}{d_{p}}\left(\frac{G C_{R M} d_{p} \rho_{\text {eff }}}{\mu_{B F}(\lambda-\gamma T)}\right)^{m}
$$

where $\lambda$ and $\gamma$ are viscosity parameter of nanofluids. The comparison between our proposed model with experimental data i.e. water-based $1 \% \mathrm{Al}_{2} \mathrm{O}_{3}$ nanofluids fraction is shown in Fig. 3. It appears that the model has a small variance. Compared to other models such as the Maxwell model and the Jang and Choi model [9] our model has a higher level of accuracy. 


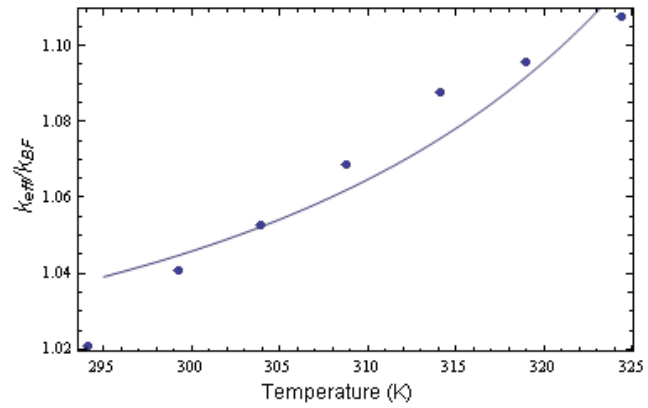

Figure 3. Comparison of conductivity model in eq. (26) with the experimental data [29] for $\mathrm{Al}_{2} \mathrm{O}_{3}$ nanofluids $(d=38.4 \mathrm{~nm}, f=$ $1 \%)$.

\section{Conclusions}

Through modification of the nanoconvection formula in the nanofluids heat transfer mechanism proposed by Jang and Choi we propose a model of thermal conductivity of nanofluids. The Reynolds number and Prandtl number are expressed as functions of the thermophysical parameters. The model is used to formulate the conductivity relationship with the volume fraction of suspended nanoparticles in nanofluids. This model allows different materials to have different thermophysical parameters. In this article we also propose a model of viscosity as a function of temperature because it can provide very significant results in nanoconvection mechanisms. The substitution of the viscosity model into the nanofluids conductivity formulation lead us to the conductivity equation as a temperature function. The accuracy of our models can be seen through comparison between the models with the experimental data.

The authors are grateful for the financial support provided by Directorate for Research and Community Service, Ministry of Research, Technology and Higher Education of the Republic of Indonesia (RISTEKDIKTI) (contract no 120.E/UN50.3.1/PM/2018).

\section{References}

[1] J. R. Lovering, A. Yip, T. Nordhaus, Energy Policy 91,517-522 (2009).

[2] J. Buongiorno \& L. Hu, Proceedings of the ASME 2009 2nd Micro/Nanoscale Heat \& Mass Transfer International Conference,517-522[ (2009).

[3] S. Kim, I. Bang, J. Buongiorno, L. Hu, International Journal of Heat and Mass Transfer 50, 4105-4116 (2007).

[4] S. You, J. Kim \& K. Kim, Applied Physics Letters 83, 3374-3376 (2003).

[5] J. Bungoiorno, L. Hu, G. Apostolakis, R. Hannink, T. Lucas \& A. Chupin, Nuclear Engineering and Design 239, 941-948 (2009).

[6] K. Wong \& O. Leon, Advances in Mechanical Engineering 2, 519659 (2015).

[7] S. Jang \& U. Choi, Applied Physics Letters 84, 4316-4318 (2004).
[8] K. Kihm, C. Chon, J. Lee \& S. Choi, Nanoscale Research Letters 6, 361 (2011).

[9] S. Jang \& S. Choi, Journal of Heat Transfer 129, 617-623 (2007).

[10] Y. Xuan \& Q. Li, J. Heat Transfer 125, 151-155 (2003).

[11] B. Pak \& Y. Cho, Exp. Heat Transfer 11,151-170, (1998).

[12] H. Zerradi, S. Ouaskit, A. Dezairi, H. Loulijat \& S. Mizani, Advanced Powder Technology 25, 11241131 (2014).

[13] N. Masoumi, N. Sohrabi \& A. Behzadmehr, Journal of Physics D: Applied Physics 42, 055501 (2009).

[14] C. Kittel \& H. Kroemer, Thermal Physics, San Fransisco: Freeman and Company, 1980.

[15] P. Keblinski, S. Phillpot, S. Choi \& J. Eastman, Int. J. Heat Mass Transfer 45, 855-863, (2002).

[16] U. Ahmad, M. Hasreen, N. Yahaya dan B. Rosnaidah, Procedia Engineering 170, 541-546, (2017).

[17] S. Kakac dan A. Pramuanjaroenkij, International Journal of Heat and Mass Transfer 52, 3187-3196, (2009).

[18] R. Brodkey, D. Kim dan W. Sidner, Int. J. Heat Mass Transfer 34, 2327-2337, (1991).

[19] R. Prasher, P. Bhattacharya dan P. Phelan, Journal of Heat Transfer 128, 588-595, (2006).

[20] Y. Xuan dan W. Roetzel, Int. J. Heat Mass Transfer 43, 3701-3707, (2000).

[21] M. Buschmann, R. Azizian, T. Kempe, J. Julia, R. Martinez-Cuenca, B. Sunden, Z. Wu, A. Seppala dan T. Ala-Nissila, International Journal of Thermal Sciences 129, 504-531, (2018).

[22] S. Tomitika, T. Aoi dan H. Yosinabu, Proc. R. Soc. London, Ser. A. 219, 233-244, (1953).

[23] M. Benissan, S. Akwaboa dan P. Mensah, Proceedings of the ASME 2012 Summer Heat Transfer Conference, 809-818, (2012).

[24] H. Mintsa, G. Roy, C. Nguyen dan D. Doucet, International Journal of Thermal Sciences 48, 363371, (2009).

[25] H. Balla, S. . Abdullah, E. A. Al-Mulla, W. Mahmood, R. Zulkifli dan K. Sopian, Res. Chem. Intermed. 39, 2197-2210, (2013).

[26] J. Buongiorno, Journal of Heat Transfer 126, 240250, (2006).

[27] C. Nguyen, F. Desgranges, G. Roy, N. Galanis, T. Mare, S. Boucher dan H. Mintsa, International Journal of Heat and Fluid Flow 28, 1492-1506, (2007).

[28] A. Einstein, Investigation on the Theory of Brownian Movement, New York: Dover, 1956.

[29] S. Das, N. Putra, P. Thiesen dan W. Roetzel, ASME J. Heat Transfer 125, 567-574, (2003). 